\title{
Chrysanthemum zawadskii extract induces hair growth by stimulating the proliferation and differentiation of hair matrix
}

\author{
ZHENG LI $^{1}$, JINGJIE LI ${ }^{1}$, LIJUAN GU ${ }^{1}$, SHAHNAZ BEGUM ${ }^{1}$, YUNBO WANG ${ }^{1}$, \\ BAISHEN SUN ${ }^{2}$, MIRA LEE $^{1}$ and CHANGKEUN SUNG ${ }^{1}$ \\ ${ }^{1}$ Department of Food Science and Technology, College of Agriculture and Biotechnology, \\ Chungnam National University, Daejeon, Chungnam 305-764, Republic of Korea; \\ ${ }^{2}$ College of Chemistry, Taizhou University, Linhai, Zhejiang 317000, P.R. China
}

Received January 29, 2014; Accepted March 29, 2014

DOI: $10.3892 / \mathrm{ijmm} .2014 .1768$

\begin{abstract}
Chrysanthemum zawadskii has been proven to possess hair growth activity and has been used as treatment for hair loss. The aim of this study was to provide a novel explanation of the mechanism by which Chrysanthemum zawadskii extracts $(\mathrm{CZe})$ promote hair growth and to characterize the affected hair follicle (HF) regions and the progression of growth. The n-butanol and water fractions of CZe were used for hair growth induction by topical application to the backs of C57BL/6 mice for up to 30 days. To investigate cell development during HF morphogenesis, bromodeoxyuridine-labeled skin sections were detected using immunohistochemistry. The results showed that the water fraction of $\mathrm{CZe}$ promoted hair shaft production and induced premature entry of telogen HFs into the anagen. Subsequently, immunohistochemical studies indicated that the water fraction of CZe stimulated the differentiation and proliferation of pluripotent epidermal matrix cells in the matrix region and epithelial stem cells in the basal layer of the epidermis. Additionally, flavonoids were identified as effective constituents. Therefore, the findings of this study suggested that the water fraction of CZe may be developed as a therapeutic agent for the prevention of hair loss.
\end{abstract}

\section{Introduction}

Chrysanthemum zawadskii var. latilobum (CZ), is an annual and perennial herb with noteworthy flowers, which has been used in traditional medicine for the treatment of inflammatory diseases, gastroenteric disorders, bladder-related disorders and hypertension (1). The effective constituents comprised

Correspondence to: Dr Changkeun Sung, Department of Food Science and Technology, College of Agriculture and Biotechnology, Chungnam National University, 220 Gung-dong, Yusung-gu, Daejeon, Chungnam 305-764, Republic of Korea

E-mail: kchsung@cnu.ac.kr

Key words: Chrysanthemum zawadskii, flavonoids, hair growth, epithelial stem cell, proliferation terpenoids and essential oils, as well as flavonoids and polysaccharides. Terpenoids and flavonoids are considered the active pharmaceutical ingredients (2), and flower extracts have been shown to have numerous pharmacological properties, including anti-allergic, anti-inflammatory and anticancer activities (3-5).

The hair follicle (HF) is a mammalian skin organ that produces hair that follows a specific growth cycle with three distinct and concurrent phases: anagen, catagen and telogen (6). Findings of previous studies on HF morphogenesis demonstrated that bulge (a niche for adult stem cells) stem cells migrate downwards and enter the matrix where they rapidly proliferate and differentiate to form HFs (7-10). During anagen, follicles are long and straight, and the proliferating matrix cells have a cycle length of $\sim 18 \mathrm{~h}$. Dead cells degenerate as they reach the upper follicle, releasing the hair shaft which then continues through the skin surface. The duration of anagen determines the hair shaft length and is dependent on the continued proliferation and differentiation of matrix cells in the matrix region of the HF (11).

Animal models comprising a variety of species, including mice, rats, sheep, rabbits and monkeys, are an important tool in the study of hair growth. However, murine models are widely used for hair growth studies due to the availability of large databases and specific mutants such as nude, hairless, rhino, and severe combined immunodeficient mice $(12,13)$. To achieve perfectly synchronized anagen induction, black pigmentation of $\mathrm{C} 57 \mathrm{BL} / 6$ or $\mathrm{C} 3 \mathrm{H}$ mice in dorsal skin is employed. This is the most commonly used site for the observation of anagen initiation as the truncal epidermis in this species lacks melanin-producing melanocytes and melanin production is strictly connected with the anagen phase of hair growth $(14,15)$.

In recent years, traditional herb medicines, such as eclipta alba, asiasari radix, panax ginseng and schisandra nigra, have been widely used for hair growth studies. Eclipta alba induced telogen to anagen transition and was positive for FGF-7 and Shh and negative for BMP4 $(12,16)$. Asiasari radix was demonstrated to stimulate hair growth in $\mathrm{C} 57 \mathrm{BL} / 6$ and $\mathrm{C} 3 \mathrm{H}$ mice by increasing the proliferation of $\mathrm{HaCaT}$ and human dermal papilla cells (DPCs) and inducing the expression of VEGF in human DPCs (17). The topical application of the schisandra nigra extract enhanced hair growth by 
increasing the expression of proliferating cell nuclear antigen (PCNA), proliferation of immortalized vibrissa dermal papilla cells, and downregulation of transforming growth factor- $\beta 2$ (TGF- $\beta 2$ ) in the bulb matrix region (18). The main molecular components in panax ginseng are ginsenosides which are known to improve cell proliferation of human DPCs through anti-apoptotic activation (19). In the present study, four fractions of CZes were used for the induction of hair growth in order to examine the mechanism of hair growth using immunohistochemistry.

\section{Materials and methods}

Extract preparation. Dried whole plants of CZ were purchased from Jecheon Medicinal Herb, Inc. (Jecheon, Korea). The dried plant of $\mathrm{CZ}$ was crushed and extracted using $70 \%$ ethanol by ultrasonic extraction for $2 \mathrm{~h}$. The filtered liquid from the extraction was concentrated in a rotary vacuum evaporator (Eyela N-N, Tokyo, Japan). The residues were then diluted with deionized water. The extracted sample was layered step by step using the dissolvents: petroleum ether, diethyl ether, and n-butanol. Following collection, concentration and quantification, petroleum ether, diethyl ether, n-butanol and water fractions were dissolved in $70 \%$ ethanol with a final concentration of 5\%. A systematic investigation of cell development on hair regeneration using water fraction was then conducted.

Experimental procedures. Eight-week-old, female C57BL/6 mice were used for all the experimental procedures. The animals were purchased from Danhan Biolink, Inc. (Eumseong, Korea). Animal experiments were conducted in accordance with the NIH guide for the care and use of laboratory animals. The animals were housed under conventional conditions with food/water available ad libitum and 12-h light cycle. Mice were divided into five groups ( $n=7$ per group). All the mice were depilated topically with depilatory cream on dorsal skin. Each mouse was administered a variety of CZe at a dose of $1,600 \mathrm{mg} / \mathrm{kg}$ body weight by daily topical application for 30 days. The conversion of each phase was recorded on a daily basis.

Bromodeoxyuridine (BrdU) labeling. BrdU is an analogue of thymidine, which is incorporated into newly synthesized DNA of replicating cells (S-phase of the cell cycle), substituting for thymidine during DNA replication. It is commonly used in the detection of proliferating and surviving cells in living tissues $(20,21)$. As a proliferative marker of adult epithelium, the timing of BrdU injection is a critical factor in differentiating newly proliferated cells from epidermal label-retaining cells (22). In order to investigate cell development in different hair growth stages and to ensure that the results reflected the true level of cell proliferation and survival stimulated by CZes, a single and a timed injection of BrdU were used for measuring cell proliferation and survival independently in different time periods (Fig. 1). To detect cell proliferation, BrdU was administered by intraperitoneal injection $(160 \mu \mathrm{g} / \mathrm{g}$ of body weight; Sigma, St. Louis, MO, USA) $24 \mathrm{~h}$ prior to mice being sacrificed at day 8,16 and 21 . To detect cell survival, the mice were injected with BrdU (160 $\mu \mathrm{g} / \mathrm{g}$ of body weight) every $12 \mathrm{~h}$ for a total of six injections prior to the 3 days of depila- tion, followed by a chase of 19 days. At day 22, mice were then sacrificed and the surviving BrdU-positive cells were detected as described in subsequent sections.

Quantification of BrdU-positive cells. The quantification and analysis of BrdU-positive cells was performed as previously described with some modifications $(23,24)$. Briefly, a random selection of seven complete HFs and equal length of epidermis tissues in each skin section was counted. The BrdU-labeled cells in the different parts of the HF were counted in each follicular section by an observer who was blinded to the study. All counts were performed at a magnification of $x 400$ and $x 1,000$ under a light microscope (Olympus BX41; Olympus, Tokyo, Japan). A cell was counted as being in the matrix region of the HF if it was in contact with or in the matrix region. The mean number of labeled cells in each part of the HF and epidermis were used for comparison.

Histology and immunohistochemistry. Paraffin-embedded skin tissues were sectioned and attached to positively charged slides. Histology was carried out using H\&E (Mayer hematoxylin and eosin; Sigma) staining. Immunohistochemical analysis of skin sections was performed as previously described (25). Briefly, the sections were deparaffinized and rehydrated in graded ethanol and placed in $100 \mathrm{mM}$ phosphatebuffered saline (PBS) for $10 \mathrm{~min}$ prior to initiating the staining procedure. The sections were then sequentially incubated with peroxidase quenching solution $\left(28 \% \mathrm{H}_{2} \mathrm{O}_{2}\right.$ : absolute pure methanol, 1:9, v:v) to eliminate the endogenous peroxidase activity for $10 \mathrm{~min}$. After incubation with the blocking solution (Life Technologies, Carlsbad, CA, USA) for $15 \mathrm{~min}$, the blotted slides (without washing) were incubated with anti-BrdU primary antibody (1:200; Santa Cruz Biotechnology, Inc., Santa Cruz, CA, USA), in a moist chamber, at room temperature (RT) for $3 \mathrm{~h}$. Subsequently, the sections were incubated with a biotinylated secondary antibody (Life Technologies) for $20 \mathrm{~min}$ at RT. After staining in 3,3'-diaminobenzidine (DAB), slides were dehydrated in a grade series of alcohol and xylene. The sections were then mounted with Histomount (Life Technologies).

Statistical analysis. Data were evaluated statistically using one-way analysis of variance or the Student's t-test. Data were expressed as the mean number of proliferating and surviving cells \pm the standard deviation. The significant difference between the values for the various experimental and control groups was set at $\mathrm{P}<0.05$.

\section{Results}

Water and n-butanol fractions of the CZe induce early onset anagen transition from telogen. Hair growth initiation time was significantly reduced following treatment with the water fraction of CZe. C57BL/6 murine dorsal hair is known to have a time-synchronized hair growth cycle and the dorsal skin pigmentation was considered as evidence for the transition of hair growth stages. Following topical application onto the backs of mice for up to 7 days, the water fraction of the $\mathrm{CZe}$ induced premature entry of telogen HFs into anagen whereas the vehicle ( $70 \%$ ethanol, control) showed no anagen induction until day 9 (Fig. 2A). The percentage of black pigmentation 
Table I. In vivo effect of Chrysanthemum zawadskii extracts on hair growth.

\begin{tabular}{|c|c|c|c|c|c|c|}
\hline \multirow[b]{2}{*}{ Measurements } & \multicolumn{3}{|c|}{ Anagen (Day 9) } & \multicolumn{3}{|c|}{ Telogen (Day 22) } \\
\hline & $\begin{array}{l}\text { N-butanol } \\
\text { fraction }\end{array}$ & $\begin{array}{l}\text { Water } \\
\text { fraction }\end{array}$ & Control & $\begin{array}{l}\text { N-butanol } \\
\text { fraction }\end{array}$ & $\begin{array}{l}\text { Water } \\
\text { fraction }\end{array}$ & Control \\
\hline Hair shaft length (mm) & - & - & - & $10.57 \pm 0.50$ & $11.37 \pm 0.55^{\mathrm{a}}$ & $10.40 \pm 0.76$ \\
\hline $\begin{array}{l}\text { Hair follicle counts } \\
\text { ( } 2.2 \mathrm{~mm} \text { longitudinal section) }\end{array}$ & $17.00 \pm 3.58$ & $15.33 \pm 2.58$ & $17.33 \pm 2.25$ & $11.37 \pm 1.77$ & $12.00 \pm 1.41^{\mathrm{b}}$ & $9.75 \pm 1.49$ \\
\hline Diameter of follicle matrix $(\mu \mathrm{m})$ & $83.00 \pm 12.90$ & $92.50 \pm 9.38^{c}$ & $81.00 \pm 8.81$ & $31.55 \pm 1.24$ & $35.45 \pm 4.66$ & $31.78 \pm 3.7$ \\
\hline Hair follicle length $(\mu \mathrm{m})$ & $779.00 \pm 86.99$ & $909.83 \pm 82.64^{\mathrm{b}}$ & $756.67 \pm 98.24$ & $234.93 \pm 13.21$ & $240.13 \pm 19.49$ & $224.15 \pm 9.63$ \\
\hline Dermis thickness $(\mu \mathrm{m})$ & $505.00 \pm 33.65$ & $513.00 \pm 53.05$ & $501.17 \pm 26.87$ & $241.87 \pm 30.31$ & $224.65 \pm 17.09$ & $276.37 \pm 14.91$ \\
\hline
\end{tabular}

Values are the mean \pm standard deviation $(\mathrm{SD}), \mathrm{n}=6 .{ }^{\mathrm{a}} \mathrm{P}<0.01,{ }^{\mathrm{b}} \mathrm{P}<0.05$ and ${ }^{\mathrm{c}} \mathrm{P}=0.053$, significance vs. control.

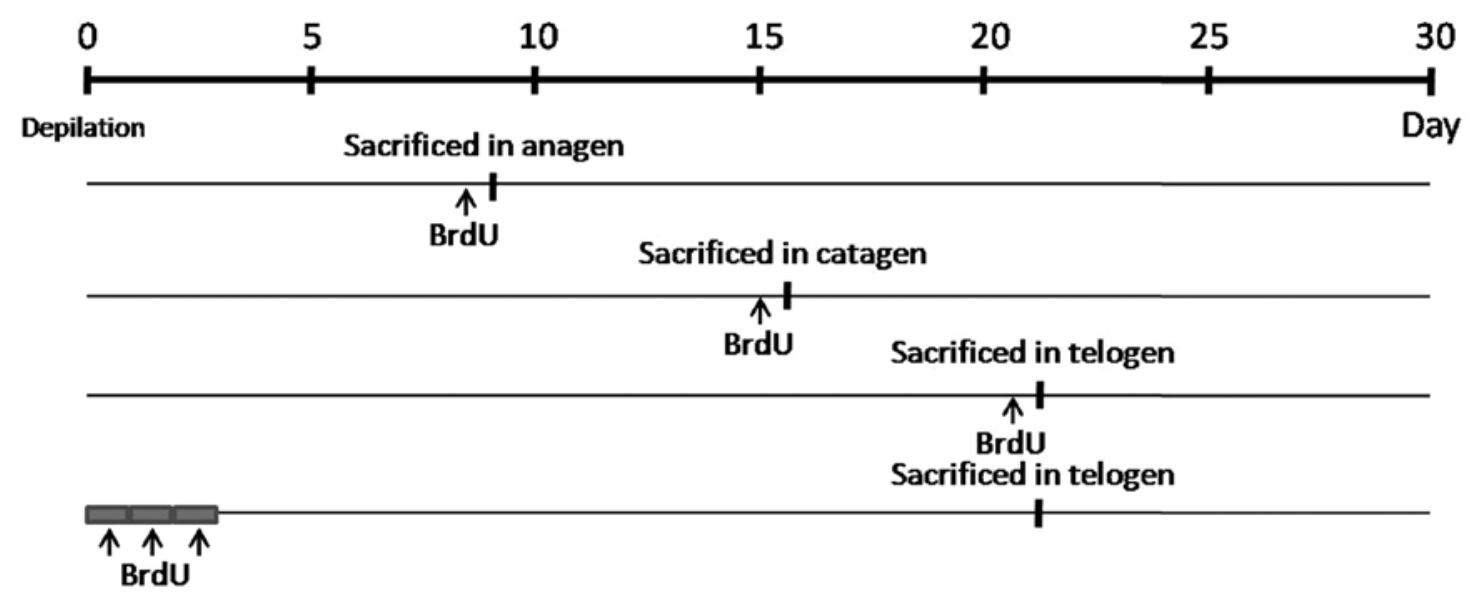

Figure 1. Experimental design for labeling of cell proliferation and survival during different phases of hair growth.

was observed during anagen-telogen development in the mice treated with CZes. These results suggested that CZes, including the n-butanol and water fractions, significantly induce anagen elongation (Fig. 2B). At day 22, the mean hair shaft length was observed in the telogen stage (Table I), which showed that the water fraction of the CZe stimulated hair fiber production. Statistical analysis demonstrated that the water fraction of the CZe markedly increased the HF size, and an increase was observed in the diameter of the matrix region $(\mathrm{P}=0.053)$ and the HF length (Table I). In addition, no inflammation, scaling or drying of skin was observed at the site of application in any of the animals during the experimental procedure. The petroleum ether and diethyl ether fractions did not show anagen induction and hair shaft elongation (data not shown).

The water fraction of the CZe causes hyperproliferation in the basal layer of the epidermis. During anagen initiation, the bulge (niche of multipotent stem cells) stem cells migrate out of the follicle to generate sebaceous glands and epidermis $(9,26,27)$. In this study, the water fraction of CZe induced a 2-fold increase in the growth of the epidermis compared to the control and n-butanol fraction groups (Fig. 3A-D). To understand whether the thickening of the epidermis was due to increased proliferation in the basal layer of the epidermis, the proliferation index of BrdU incorporated by using immunohistochemistry at day 9 was measured. The results indicated that the water fraction of the CZe increased proliferation in the basal layer of the epidermis (Fig. 3E-H).

The water fraction of the CZe increases cell proliferation in anagen and telogen of matrix HFs. Cell proliferation and differentiation in the matrix is required for HF formation. Pluripotent epidermal matrix cells, which have dermal papilla at their base, in the hair bulb move upward while cells at the center of the follicle become the medulla of the hair shaft $(26,28)$. To investigate whether the increase of the hair shaft occurred to hyperproliferation in the matrix region, we traced the cell development of hair matrix during the anagen to telogen transition. The results suggested that the water fraction of the CZe markedly increased cell proliferation during the anagen phase of hair matrix (Fig. 4E-H). Additionally, the quantity of proliferating $\mathrm{BrdU}^{+}$cells in telogen demonstrated that the activation of hyperproliferation in the matrix was maintained in the telogen stage (Fig. 5I-L). Moreover, when the quantification of cell proliferation and survival in the epidermis was performed at day 22 , the statistical index showed that the water fraction of CZe stimulated proliferation in the basal layer of the epidermis and not only in 
A

Day 7
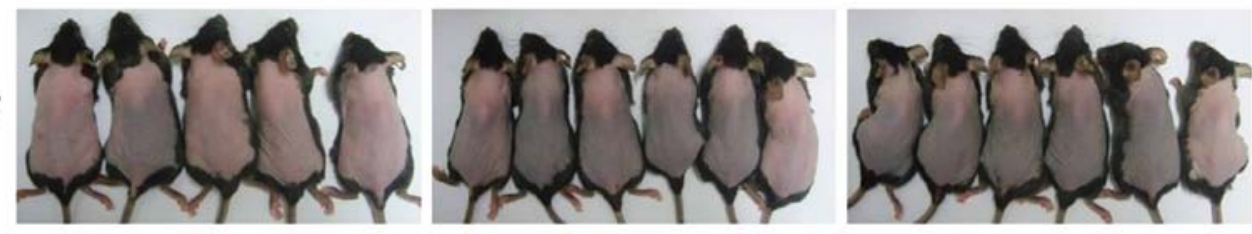

Day 9



Control

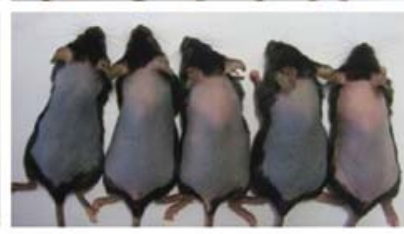

$\mathrm{N}$-butanol fraction

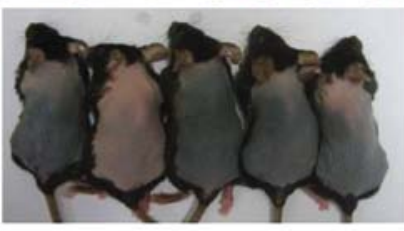

Water fraction

B

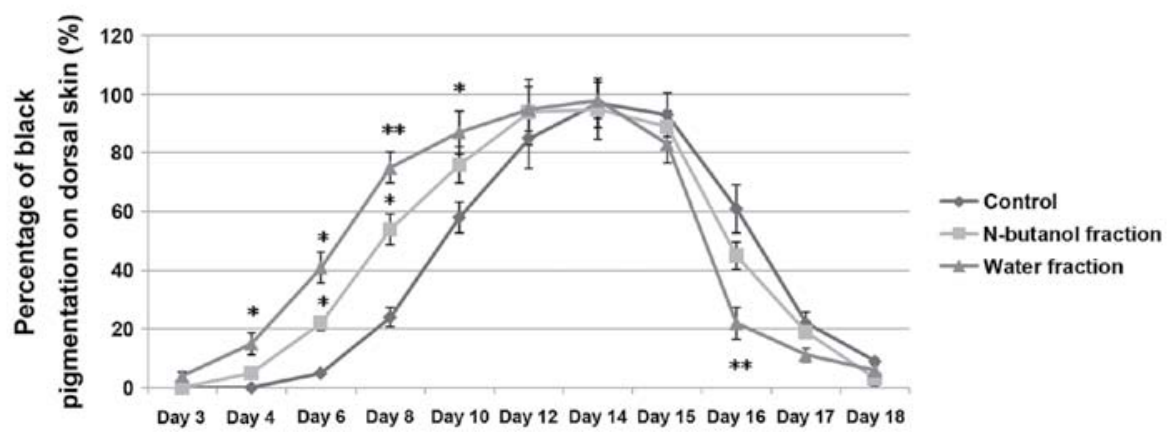

Figure 2. N-butanol and water fractions of Chrysanthemum zawadskii extracts (CZe) induced premature anagen transition. (A) Daily topical application for 7 and 9 days after depilation. Note the daily hair growth observed in the CZe-treated group. (B) The ratio of the anagen phase on dorsal skin at days 8 - 12 . ${ }^{*} \mathrm{P}<0.05,{ }^{* *} \mathrm{P}<0.01$ vs. the control.

anagen (Fig. 5A-D), increasing the number of surviving cells (Fig. 5E-H). In addition, the n-butanol and water fractions of the CZe had no effect on proliferation in the mid-section of the HFs (Fig. 4A-D) and no effect on cell survival in the telogen stage of HF (data not shown).

\section{Discussion}

In the present study, we have shown that when the shaved skin of C57BL/6 mice is treated with topical application of water and n-butanol fractions of CZe for 25 days, new hair morphogenesis is initiated in the shaved area, with the initiation time for this process to occur also being significantly reduced when compared to vehicle-treated mice. In order to elucidate the biological mechanism by which CZes induce early onset anagen transition; we investigated cell development in full-thickness skin by using BrdU incorporation. The results indicate that the water fraction of the CZe promoted cell proliferation in the basal layer of the epidermis and induced the proliferation and differentiation of epidermal matrix cells in anagen and telogen of HFs. During anagen, the new HF morphogenesis was initiated by a number of pluripotent stem cells that are normally present in the bulge region, including epithelial stem cells. These stem cells migrate downwards into the regenerating epithelium where they can differentiate into at least eight different cell lines, forming the ORS, companion layer, Henle's layer, Huxley's layer, cuticle of the IRS, cuticle of the hair shaft, shaft cortex and shaft medulla $(29,30)$. It is also known that the lower region of the whisker follicle contains cells that respond to skin morphogenetic signals by upward and downward migration and form epidermis, HFs and sebaceous glands $(9,31)$. Thus, we hypothesized that the hyperproliferation in the basal layer of the epidermis may occur via stimulation of the migration and differentiation of epithelial stem cells during anagen initiation, and that the premature entry of telogen HFs into anagen may occur through the induction of epithelial stem cell downward migration and differentiation to form the new dermal papilla and hair germ. In addition, stimulation of differentiation and proliferation of pluripotent epidermal matrix cells in the matrix region may be one of the mechanisms by which the water fraction of CZes increase the hair shaft length and the HF size.

Previously, we had performed the screening test by using crude extracts of herb medicines $(17,18)$. The results indicated that $\mathrm{ZC}$ crude extract showed a marked induction on anagen initiation. To investigate the effective constituent on hair growth in $\mathrm{CZ}$ crude extract, the petroleum ether, diethyl ether, $\mathrm{n}$-butanol and water fractions of CZe, were used in the present study. Petroleum ether and diethyl ether fractions did not show induction on anagen initiation and hair shaft production. Thus, liposoluble terpenoids, sterols and essential oils, as the main components, did not stimulate hair growth. By contrast, flavonoids, the main bioactive constituents in n-butanol and water fractions, were considered as effective constituents. Notably, quercetin, luteolin and linarin, the main flavonoids in CZe, should be studied in future studies to determine whether they serve as potential effective compounds.

HF is a mammalian skin organ that produces hair in a process that occurs in phases, including the growth, regression and resting phases. The migration and differentiation of epithelial stem cells is principally responsible for hair production $(27,32)$. Anagen is the active growth phase responsible for 

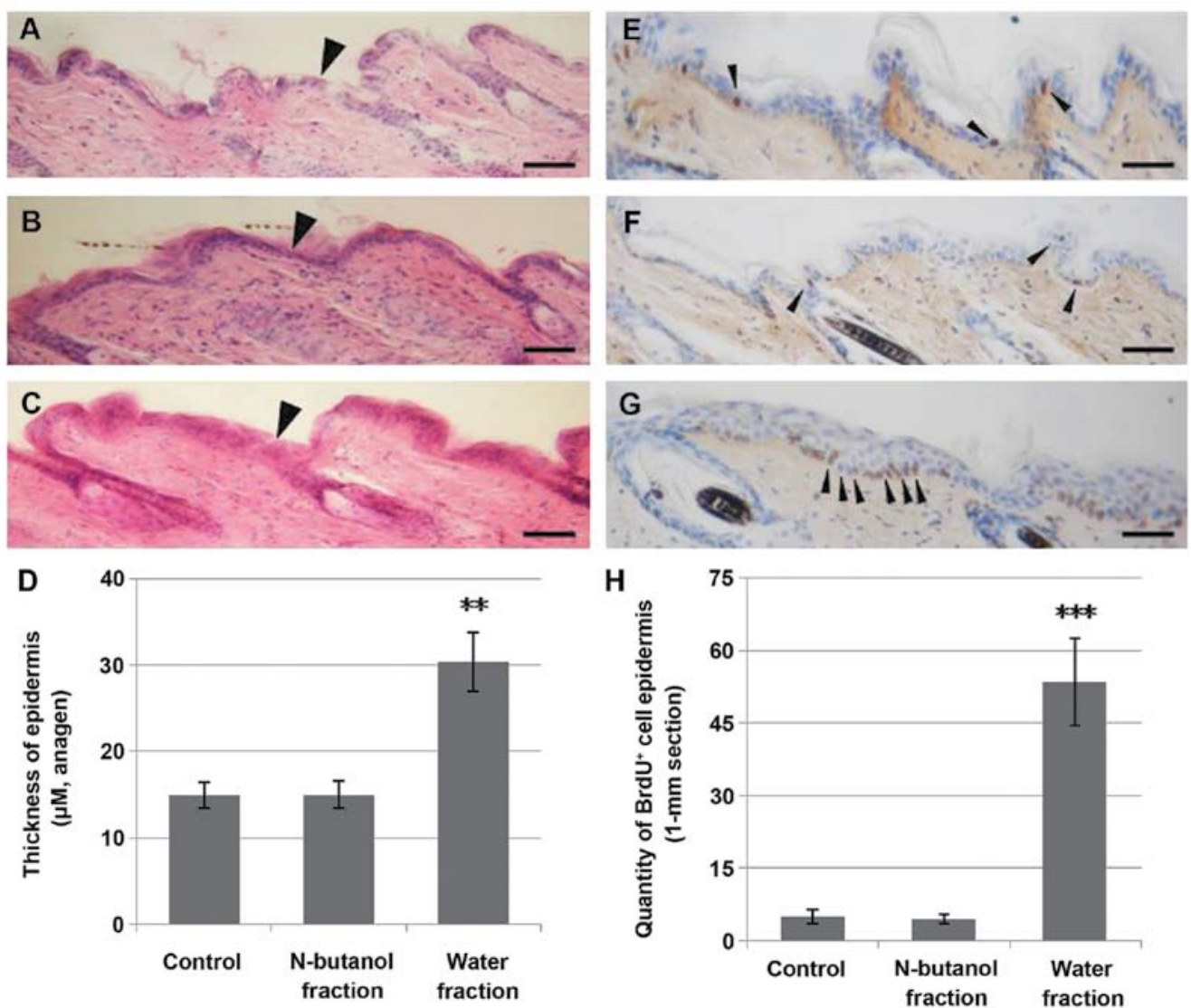

Figure 3. Histological analysis and quantification of proliferating bromodeoxyuridine (BrdU') cells in the basal layer of the epidermis. (A-C) Hematoxylin and eosin (H\&E) staining of the epidermis. (A) Control, (B) n-butanol fraction and (C) water fraction. (D) Induction of epidermal thickness following exposure to the water fraction of CZ. The thickness of the epidermis in mice exposed to the water fraction of CZ is 2-fold greater than that observed in the control and the n-butanol fraction. An example, at day 9, is the labeled retaining cells in the basal layer of the epidermis. (E) Control, (F) n-butanol fraction, (G) water fraction. (H) Quantification of proliferating cells after $24 \mathrm{~h}$. Scale bar, $50 \mu \mathrm{m}$. Black arrows indicate (A-C) the change of the epidermis, and (E-G) the labeling of proliferated cells in the basal layer of the epidermis. ${ }^{* *} \mathrm{P}<0.01,{ }^{* * *} \mathrm{P}<0.001$ vs. control.
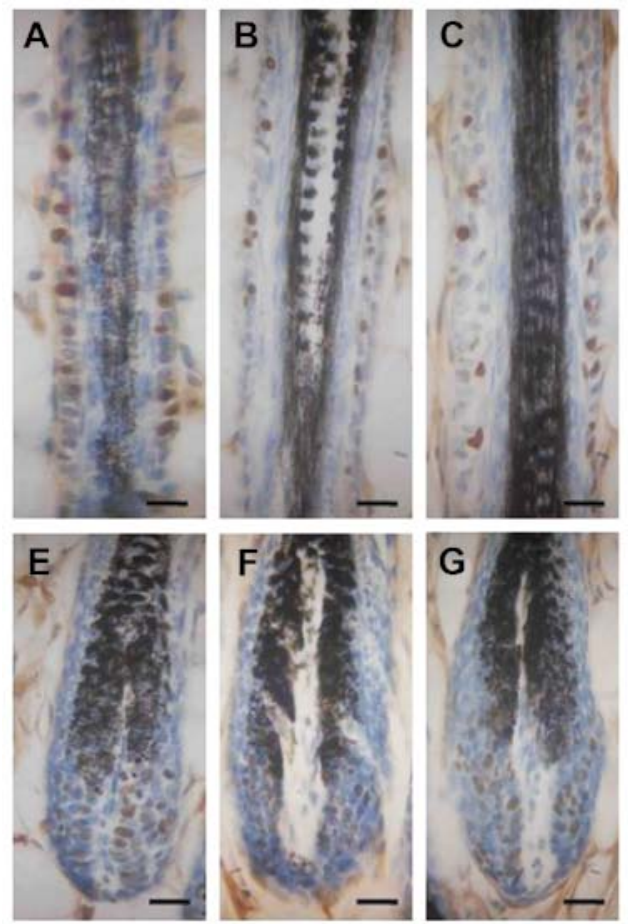

D

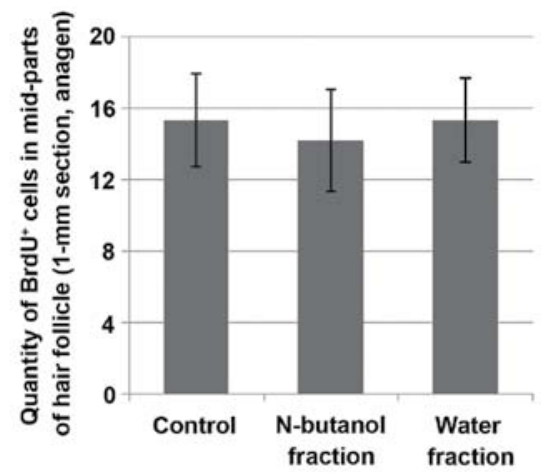

H

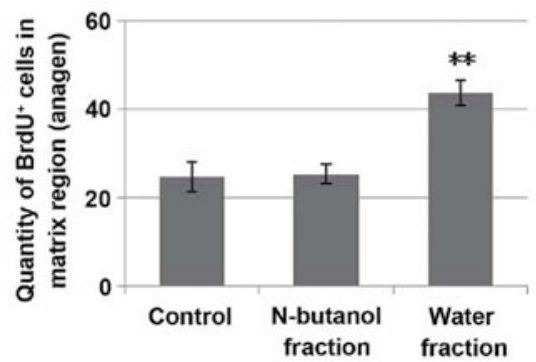

Figure 4. Immunohistochemical analysis of bromodeoxyuridine $\left(\mathrm{BrdU}^{+}\right)$retention in anagen of hair follicles. (A-C) Location of proliferating cells in the mid-sections of the hair follicles. (A) Control, (B) n-butanol fraction and (C) water fraction. (D) Quantification of proliferated cells after $24 \mathrm{~h}$. (E-F) Location of proliferated cells in hair matrix. (E) Control, (F) n-butanol fraction and $(\mathrm{G})$ water fraction. $(\mathrm{H})$ The water fraction induced hyperproliferation in the hair matrix. Scale bar, $25 \mu \mathrm{m} .{ }^{* *} \mathrm{P}<0.01$ vs. control. 


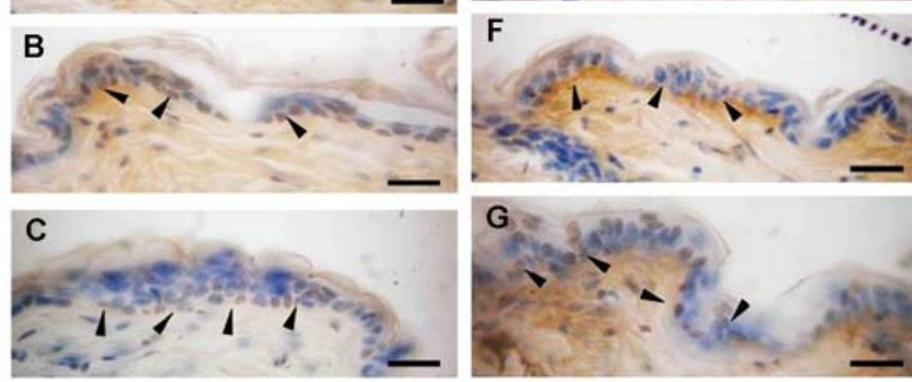

H
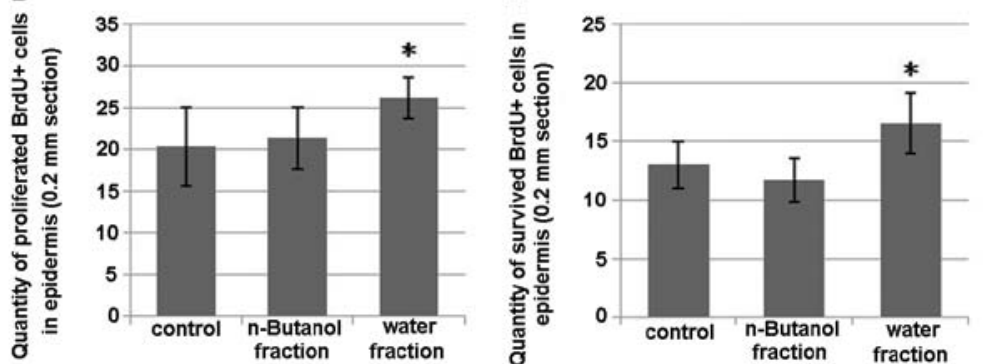

L

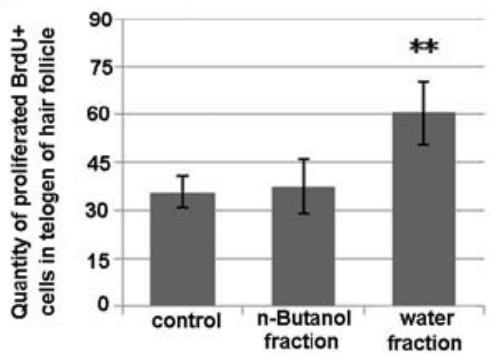

Figure 5. Immunohistochemical analysis of bromodeoxyuridine $\left(\mathrm{BrdU}^{+}\right)$cells retained in telogen at day 22. (A-C) Proliferating BrdU ${ }^{+}$cells in the basal layer of the epidermis. (A) Control; (B) n-butanol fraction; and (C) water fraction. (D) The water fraction of Chrysanthemum zawadskii extracts (CZe) stimulates cell proliferation in telogen epidermis within $24 \mathrm{~h}$ as shown by the quantification of surviving cells in the epidermis. (E) Control; (F) $\mathrm{n}$-butanol fraction; and ( $\mathrm{g}$ ) water fraction. $(\mathrm{H})$ The water fraction of CZe increases the number of surviving cells, and elongate the cell life-span. (J-K) Location of proliferating BrdU ${ }^{+}$cells in telogen hair follicles. (I) Control, (J) n-butanol fraction, and (K) water fraction. UF, upper follicle; SG, sebaceous gland; M, matrix; SHG, secondary hair germ. Red arrows show the cell proliferation in SG. Black arrows indicate the labeling of proliferated and survived cells in the epidermis and hair follicles. (L) Quantity of proliferating BrdU ${ }^{+}$cells in the telogen of hair follicles. Scale bar, $25 \mu \mathrm{m}$. ${ }^{*} \mathrm{P}<0.05 ;{ }^{* *} \mathrm{P}<0.01$ vs. control.

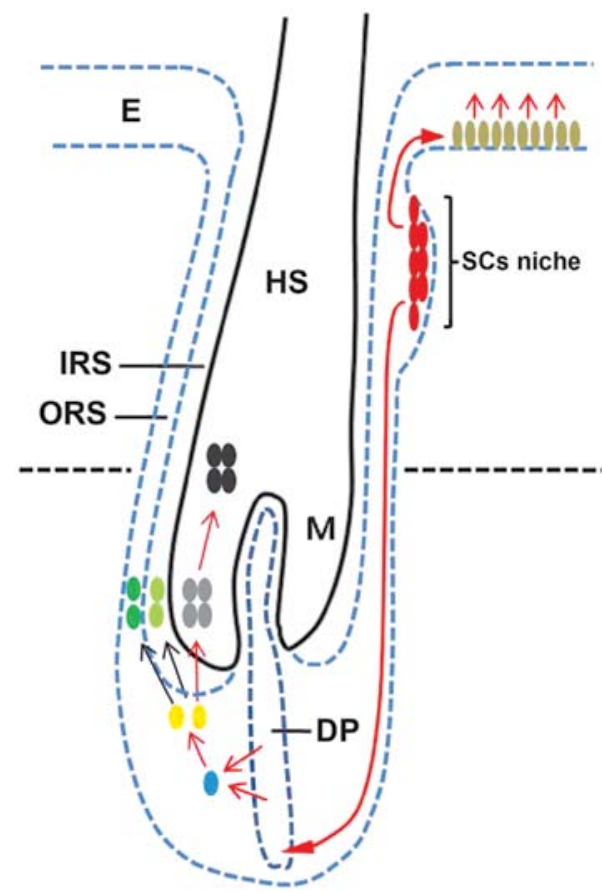

Figure 6. The proposed mechanism for Chrysanthemum zawadskii regulation of anagen initiation and hair shaft production. i) Anagen initiation leads to the induction of bulge stem cell migration up- and downward, with stem cells differentiating to form the epidermis and new hair germ. ii) Stimulation of epidermal matrix cell proliferation and differentiation. iii) Promotion of the differentiation of adult progenitor and precursor cells to form the hair shaft. Red arrows indicate the potential pathway; black arrows indicate the hypothetical pathway. forming the HF and hair fiber. During this phase the roots of the hair divide rapidly and add to the hair shaft (32). To understand the mechanism of CZe stimulation of hair shaft production, we labeled the cell proliferation in the different time-points and noted the residence time of anagen. The results demonstrate that stimulation of differentiation in hair matrix is probably initiated by the water fraction of the CZe. The appearance of black pigmentation was taken as the anagen initiation (33). Thus, the premature entry into anagen was considered as an important symbol of migration and differentiation of epithelial stem cells. Following treatment with the water fraction of the $\mathrm{CZe}$, the epithelial stem cells were activated and migrated upward and downward, subsequently differentiating rapidly to form the interfollicular epidermis and new HF during anagen phase. In consideration of these findings, we suggest the hypothetical model shown in Fig. 6 (red arrows show the potential pathway).

Hair cycle is regulated by the interplay of stimulatory and inhibitory growth factors in mice and humans (6). Previous studies on the induction of hair growth as a result of $\mathrm{CZ}$ application, reported that CZes promote proliferation of a human keratinocyte cell line and human dermal papilla cells in vitro, and subsequently, suggested that jaceosidin and eupatilin, which are both derived from $\mathrm{CZ}$, had the potential to promote hair growth through the downregulation of TGF- $\beta$ by the jaceosidin-dependent upregulation of VEGF and Bcl-2 upregulation by eupatilin (34). In the present study, we did not investigate the signaling pathways involved but performed a 
novel evaluation in order to clarify the mechanism by which CZes promote hair growth using cell labeling and tracing. It provided a more precise measure of which fraction in CZes is responsible for hair growth and may therefore provide a more reliable molecular basis for potential clinical application.

In conclusion, as a traditional herb used as medicine, Chrysanthemum zawadskii may have wide potential in clinical application. It has few side effects; no local inflammation, pruritus, edema, or scaling of skin were observed during the application procedure. The present study has located the effective region of hair growth, identified a more accurate timeline by which growth occurs and provided a novel explanation of the mechanism by which CZes promote hair growth through the regulation of cell development at different time periods. Further analysis of these results as well as a more detailed evaluation of the molecular mechanism by which CZes induce proliferation of hair growth are required before its potential can be completely realized in the clinic.

\section{Acknowledgements}

The authors would like to thank Dr Kang-Ju Choi for guidance in the process of herb extract and isolation. This study was supported by the DBIO Science Food and Technology Corporation.

\section{References}

1. Han S, Sung KH, Yim D, et al: The effect of linarin on LPS-induced cytokine production and nitric oxide inhibition in murine macrophages cell line RAW264.7. Arch Pharm Res 25: 170-177, 2002 .

2. Shim SY, Kang HS, Sun HJ, et al: Isolation and identification of flavonoids from Gujeolcho (Chrysanthemum zawadskii var. latilobum) as inhibitor of histamine release. Food Sci Biotechnol 21: 613-617, 2012.

3. Seo JY, Lim SS, Park J, et al: Protection by Chrysanthemum zawadskii extract from liver damage of mice caused by carbon tetrachloride is maybe mediated by modulation of QR activity. Nutr Res Pract 4: 93-98, 2010.

4. Singh RP, Agrawal P, Yim D, Agarwal C and Agarwal R: Acacetin inhibits cell growth and cell cycle progression, and induces apoptosis in human prostate cancer cells: structure-activity relationship with linarin and linarin acetate. Carcinogenesis 26 845-854, 2005.

5. Hsu YL, Kuo PL and Lin CC: Acacetin inhibits the proliferation of Hep G2 by blocking cell cycle progression and inducing apoptosis. Biochem Pharmacol 67: 823-829, 2004.

6. Stenn KS and Paus R: Controls of hair follicle cycling. Physiol Rev 81: 449-494, 2001.

7. Li LH and Xie T: Stem cell niche: Structure and function. Annu Rev Cell Dev Biol 21: 605-631, 2005.

8. Blanpain C and Fuchs E: Epidermal stem cells of the skin. Annu Rev Cell Dev Biol 22: 339-373, 2006.

9. Gambardella L and Barrandon Y: The multifaceted adult epidermal stem cell. Curr Opin Cell Biol 15: 771-777, 2003.

10. Reya $\mathrm{T}$ and Clevers H: Wnt signalling in stem cells and cancer. Nature 434: 843-850, 2005.

11. Alonso L and Fuchs E: The hair cycle. J Cell Sci 119: 391-393, 2006.
12. Datta K, Singh AT, Mukherjee A, Bhat B, Ramesh B and Burman AC: Eclipta alba extract with potential for hair growth promoting activity. J Ethnopharmacol 124: 450-456, 2009.

13. Sundberg JP and King LE Jr: Mouse models for the study of human hair loss. Dermatol Clin 14: 619-632, 1996.

14. Slominski A, Paus R and Costantino R: Differential expression and activity of melanogenesis-related proteins during induced hair growth in mice. J Invest Dermatol 96: 172-179, 1991.

15. Slominski A, Paus R, Plonka P, et al: Melanogenesis during the anagen-catagen-telogen transformation of the murine hair cycle. J Invest Dermatol 102: 862-869, 1994.

16. Roy RK, Thakur M and Dixit VK: Hair growth promoting activity of Eclipta alba in male albino rats. Arch Dermatol Res 300: 357-364, 2008.

17. Rho SS, Park SJ, Hwang SL, et al: The hair growth promoting effect of Asiasari radix extract and its molecular regulation. J Dermatol Sci 38: 89-97, 2005.

18. Kang JI, Kim SC, Hyun JH, et al: Promotion effect of Schisandra nigra on the growth of hair. Eur J Dermatol 19: 119-125, 2009.

19. Kim JH, Yi SM, Choi JE and Son SW: Study of the efficacy of Korean red ginseng in the treatment of androgenic alopecia J Ginseng Res 33: 223-228, 2009.

20. Konishi T, Takeyasu A, Natsume T, Furusawa Y and Hieda K: Visualization of heavy ion tracks by labeling 3'-OH termini of induced DNA strand breaks. J Radiat Res 52: 433-440, 2011.

21. Smith PJ, Howes EA and Treherne JE: Cell proliferation in the repairing adult insect central nervous system: incorporation of the thymidine analogue 5-bromo-2-deoxyuridine in vivo. J Cell Sci 95: 599-604, 1990

22. Nijhof JG, van Pelt C, Mulder AA, Mitchell DL, Mullenders LH and de Gruijl FR: Epidermal stem and progenitor cells in murine epidermis accumulate UV damage despite NER proficiency. Carcinogenesis 28: 792-800, 2007.

23. Blanpain C, Lowry WE, Geoghegan A, Polak L and Fuchs E: Self-renewal, multipotency, and the existence of two cell populations within an epithelial stem cell niche. Cell 118: 635-648, 2004.

24. Sarin KY, Cheung P, Gilison D, et al: Conditional telomerase induction causes proliferation of hair follicle stem cells. Nature 436: 1048-1052, 2005.

25. Liu L, Hoang-Gia T, Wu H, et al: Ginsenoside Rb1 improves spatial learning and memory by regulation of cell genesis in the hippocampal subregions of rats. Brain Res 1382: 147-154, 2011.

26. Alonso L and Fuchs E: Stem cells of the skin epithelium. Proc Natl Acad Sci USA 100: 11830-11835, 2003.

27. Cotsarelis G: Epithelial stem cells: a folliculocentric view. J Invest Dermatol 126: 1459-1468, 2006.

28. Spradling A, Drummond-Barbosa D and Kai T: Stem cells find their niche. Nature 414: 98-104, 2001.

29. Krause K and Foitzik K: Biology of the hair follicle: the basics. Semin Cutan Med Surg 25: 2-10, 2006.

30. Reynolds AJ and Jahoda CA: Hair follicle stem cells? A distinct germinative epidermal cell population is activated in vitro by the presence of hair dermal papilla cells. J Cell Sci 99: 373-385, 1991.

31. Oshima H, Rochat A, Kedzia C, Kobayashi K and Barrandon Y: Morphogenesis and renewal of hair follicles from adult multipotent stem cells. Cell 104: 233-245, 2001.

32. Paus R and Cotsarelis G: The biology of hair follicles. N Engl J Med 341: 491-497, 1999.

33. Peters EMJ, Botchkarev VA, Müller-Röver S, Moll I, Rice FL and Paus R: Developmental timing of hair follicle and dorsal skin innervation in mice. J Comp Neurol 448: 28-52, 2002.

34. Pi LQ, Jin XH,Kim K, et al: Effects of Chrysanthemum zawadskii extract on hair growth in vivo and in vitro. Exp Dermatol 19: 582, 2010. 